\title{
There is need in new generation smart grid for the space and ground energy systems
}

\author{
Alexander Ageev ${ }^{1}$, Svetlana Bortalevich ${ }^{2}$, Evgeny Loginov ${ }^{2, *}$, Alexander Shkuta $^{3}$, and \\ Dmitry Sorokin ${ }^{2}$ \\ ${ }^{1}$ RAS, Institute of Economic Strategies, 101000 Moscow, Russia \\ ${ }^{2}$ RAS, Institute of Market Problems, 117418 Moscow, Russia \\ ${ }^{3}$ Financial University under the Government of the Russian Federation, World \\ Economics and International Business Department, 125993 Moscow, Russia
}

\begin{abstract}
The aim of the article is to considerate the opportunities of synchronization of the space and ground systems that generate and transfer energy on the basis of new generation smart grid using. The authors substantiate the necessity of new intellectual monitoring services that assess the processes took place in "generation-transportation-distributionconsumption" space and ground systems. This is made in order to improve the dynamic indicators of the energy system and to avoid the emergencies. The authors also give a prognosis of the dynamic indicators of the electric power super-system in analyzing metastable conditions in different energy modes.
\end{abstract}

\section{Introduction}

The problem of the space and ground systems integration and electric power transfer as part of integrated super-system is high relevant nowadays. Especially it is because of the projects of the energy sources transferring from the Earth to space [1].

However, this problem may be solved very soon due to the modern technological progress.

Russia and other counties have archived some progress in creating the railguns and almost can construct the electromagnetic driver that is able to make the little objects (less than 1 kilo) have the orbital velocity and transfer goods to the orbit.

Under our project, the railguns should be placed near the Nuclear Power Station and the Hydroelectric power station, which allows to reduce the launch costs as it can use the excess of generated electricity (up to $30 \%$ at night).

\section{Problem statement}

We offer the technical and economic model under which the space power plants should be consisted of a number of standard modules that are launched through the railguns. This

\footnotetext{
* Corresponding author: loginovel@mail.ru
} 
model defines the perspective integration of both space and ground electrical engineering as one super-system.

We also want to highlight the need for the package of monitoring methods based on new generation smart grid that provides receiving the information about the processes occurred in the energy systems [2]. This of monitoring methods assumes the possibility of analyzing the interactions between various subsystems of the integrated power supersystem.

Limited transfer of energy from the orbit to the Earth provokes the lack of active power in many regions of Russia [3, 4].

The variety of manifestations of the effect of resonant stimulation (coherent resonance interaction) - from the spaced-coherent level to the integral system-structural reactions, on the one hand, and the loss of power objects from synchronism - the process of the output of the integrated power supersystem beyond the limits of the quasistable states.

On the other hand, with inevitability requires the construction of a single technical concept of self-balancing an integrated electric power supersystem.

The attempts to establish the correspondence between the stability of the vibrational levels of the "generation-transport-distribution-consumption" chain to achieve the local equilibrium point of the electric power super-system, determined by a combination of individual aperiodic or vibrational disturbances, did not lead to positive results, which, apparently, is due to insufficient consideration of the dependence on heterogeneity of the state parameters of the system objects, including a linear or nonlinear component that varies with time.

\section{Solution}

In the integrated electric power supersystem, when interacting with coherent oscillations generated by a substantially nonequilibrium active medium of separate segments of power grids and power energy objects with a large number of intelligent devices (with an inverse characteristic of the stability of the vibrational levels), self-balancing processes can occur, as a result which, in particular, will restore the functions of the damaged power soup a system that includes space and terrestrial systems.

The electric power supersystem, including space and terrestrial systems, is not only open, but also consists of active small objects, the structure of which is complex and insufficiently manageable.

Apparently, the concretization of the concept of an element of an integrated electric power supersystem essentially depends on the formulation of the problem and requires the consideration of collective interactions in the processes of energy transfer from different points of the geostationary orbits of space solar power stations to the ground.

The complex and non-linear dynamic behavior and incompleteness of knowledge about microstates in individual systems of the integrated electric power super-system make the application of coherent-resonant description unavoidable.

The possibility of using the effect of coherent resonance interaction of a quasiintegrated segment of the network (cloud, swarm) of standard modules as elements of space power plants as elementary entities is confirmed by the fact that the behavior of energy consumers and the need to take into account the influence of boundary conditions (contours supersystem) on the spectrum of admissible resonant states of a quasi-integrated segment in the chain of space and terrestrial systems "generation-transport-races limit-consumption ".

The presence of hierarchy in a multilevel structural organization of integrated space and terrestrial electric power industry as a single super system indicates the possibility of interconversion of dynamic synchronization areas (dissynchronization) of self-oscillations, as well as areas of technical activity in which stable and unstable cycles coexist. 
Such transformations can be described taking into account the properties of the scale similarity between space and terrestrial energy systems.

Another aspect of the coherent-resonance interaction effect related to the phenomena of self-organization of integrated space and terrestrial electric power industry as a single super system is the adjustment of the spatiotemporal structure of dynamically changing equilibrium parameters to the generation-transport-distribution-consumption structure determined by a combination of individual aperiodic or vibrational disturbances, occurring due to the resonant properties of the object-technical structure.

Essential in this case is the fact that the resonant interaction formed in the structure of the "generation-transport-distribution-consumption" chain determined by a combination of individual aperiodic or vibrational disturbances is practically independent of the initial structure of the energy dynamics.

The attempts to establish the correspondence between the stability of the vibrational levels of the "generation-transportation-distribution-consumption" chain to find the local equilibrium point of a non-linear electric power supersystem including space and terrestrial systems did not lead to positive results, which, apparently, is due to insufficient consideration of the degree of "openness" integrated space and terrestrial electric power generation in the process of reactive power production / consumption both in power grids and in other segments integrated power supersystem and consumer space.

The changes in the resonant state of the quasi-integrated network segment (clouds, swarms) of standard modules as elements of space power plants, for example, their "clotting" or deployment, require calculating the parameters of reaching the local equilibrium point of a nonlinear super energy system, including space and terrestrial systems, and then finding the optimization maximum of this equilibrium state from the averaged parameters of the group of interacting terrestrial and cosmic systems, but lead to significant variations in their resonant activity.

The limited resource of the quasi-integrated segment of the network (clouds, swarms) of standard modules as elements of space power stations within the super system and the need to maintain objects sufficient for life in a given resonance state lead to the emergence during the evolution of several dynamic regions of synchronization (dissynchronization) of self-oscillations, in which a stable and unstable cycle coexist.

From the point of view of the super-system, this mechanism can be considered as a system of "synchronization (dissynchronization) of self-oscillations", which causes coherent-resonant nonequilibrium of the entire electric power supersystem, including space and terrestrial systems.

From a coherent-resonant point of view, such a disequilibrium can be considered as the cause of the coherent state in the chain of space and terrestrial "generation-transportdistribution-consumption" systems and interactions of various subsystems of the integrated electric power super-system and their ensembles, synchronization and suppression of some vibrational modes by others.

The main technical characteristic of the quasi-integrated segment in the chain of space and ground systems "generation-transportation-distribution-consumption", associated with its resonance state, can be considered self-oscillating moment.

The quasi-integrated segment in the chain of space and terrestrial systems "generationtransport-distribution-consumption" interacts with the nonlinear dynamical effect of energy quasi-harmonic oscillations, changing its stability to the vibrational state due to a change in the orientation and magnitude of its self-oscillating moment.

It should be noted that the self-oscillatory (multicyclic) moment of the quasi-integrated segment of the network (clouds, swarms) of standard modules as elements of cosmic power stations or its almost independent regions is generally nonzero, if only because of the presence of quasi-coherent technical fluctuations. 
Since any technical system tends to minimize its potential energy, it can be assumed that the final effect of any coherent reaction must have a minimum self-oscillating moment of its own, if only because of the interaction of external and internal cycles of non-zero magnitude.

Therefore, in the process of coherent resonance reactions, objects have different selfoscillating moments and the super-system control operators can control their behavior and, at the end of the reaction, they mutually compensate as much as possible.

The self-oscillatory interaction of objects at resonance ensures the instability of the medium and the object, and also provides their interaction with coherent-resonant clusters of interacting factors of energy dynamics that is more effective in comparison with the final effect, which leads to stimulation of various resonance reactions.

This can be represented as follows: as a result of the self-oscillatory interaction of the quasi-integrated segment in the chain of space and terrestrial "generation-transportdistribution-consumption" systems with clusters of interacting factors, its oscillatory dynamics increases, which leads to an intensification of the process of "sorting out" the various resonant states and more rapid "finding" of a resonant state that is unstable for another object.

The disruption of the functioning of the super-system, apparently, primarily causes the accumulation of unnecessary effects of technical reactions.

Therefore, the growth of the self-oscillating moment in the objects leads to an increase in the system coherent resonance. In other words, the synchronization of the induced negative oscillations destabilizes the system or its segment and ensures the selectivity of the coherent-resonance interaction.

This simple technical consideration explains, in addition to the increased efficiency of the coherent-resonant interaction process for the unstable segments of the "generationtransport-distribution-consumption" chain, and some of the causes that lead to a difference in energy effects under conditions with a momentum "inside the system" and with a pulse "outward" system ".

Numerous data on the resonant stimulation of various effects also seem to be associated with a similar flattening of the spatial structure of the quasi- integrated segment of the network (cloud, swarm) of standard modules as elements of cosmic power stations, since the resonance center of many effects is also in the depth of a rather narrow segment.

The threshold value of the intensity of the energy dynamics, which still induces a coherent-resonant interaction, should be comparable with the degree of coherence of the nonlinear dynamic interaction of external and internal vibrational modes.

\section{Conclusion}

Elements of the electric power supersystem, including space and terrestrial systems, operate in the presence of multifactorial nonlinear dynamic interaction of external and internal cycles. Without specifying the control mechanisms of technical processes due to the objecttechnical coherent-resonant effects, it can be assumed that the multifactorial nonlineardynamic interaction determines the magnitude of the oscillations for such control systems.

The consideration of the complex analysis of data allows us to reveal the reasons of the massive dynamic disturbances in the super-system.

On this basis we can identify the self-balanced condition of the integrated power supersystem and outline the functioning dynamics of the power subsystems.

Thus, clear understanding of the reasons and identifying the steps of the massive dynamic disturbances allows us to reveal the settings of the emergency control devices that will monitor the smart grid work if it faces some problems. 
The theses were prepared with the financial support of the Russian Humanitarian Scientific Foundation (project No. 16-02-00463 a "Formation of organizational mechanisms for operating oil and gas resources on the basis of multi-agent modeling to protect Russia's economic interests from manipulating oil prices on world markets").

\section{References}

1. A. A. Makarov, T. A. Mitrova, F. V. Veselov, A. A. Galkina, V. A. Kulagin, Therm. Eng. 64(10), 703 (2017)

2. A. S. Bugaev, E. L. Loginov, A. N. Raikov, V. N. Saraev, S\&T. Inf. Pr. 36(1), 68 (2009)

3. A. S Asochakov, Y. V. Shulgina, A. I. Soldatov, E. M. Shulgin, D. N. Ogorodnikov MEACS, (2015)

4. S.V.Chubov, A.I.Soldatov IOP Conf. Ser., MEACS 177(1), (2016) 